\title{
The Assessment of Developed Mental Stress Elicitation Protocol Based on Heart Rate and EEG Signals
}

\author{
Saidatul A., Paulraj Murugesa Pandiyan, and Sazali Yaacob
}

\begin{abstract}
Nowadays, stress is one of the major issues where too much stress may lead to the depression, fatigue and insomnia. Stress can be divided into two types called Eustress and Distress. However, Distress is highly focuses research field due to the harm affect to the body system. The aim of this study is to assess the developed mental stress elicitation protocol via the physiological changes in heart rate and brain activity. It is well known that mental stress may influences the heart rate and the Electroencephalography (EEG) signal. EEG signal contains information that represents the brain state and other neurological disease. A new developed mental stress elicitation experimental protocol is proposed to trigger the mental stress via Mental Arithmetic Task (MAT). Some modifications of the existing MAT have been made improve the ability of new protocol in order to ensure the targeted level of stress is properly induced. MAT which is on dynamical excitation concept and time pressure are the two combination of stressor which were introduced in this study. EEG signal processing such as preprocessing, feature extraction and classification were employed to extract and classified the mental stress features. In order to verify the ability of proposed mental stress elicitation protocol to induce properly as targeted level, three validation methods i.e statistical analysis (paired t-test), k-nearest neighbors (kNN) and Alpha Brain Asymmetry Score were adapted. Based on the results from these three validation methods, it was found that the proposed mental stress elicitation protocol is comparable to be used for further mental stress study.
\end{abstract}

Index Terms-Electroencephalography (EEG), heart rate mental stress, mental arithmetic task (MAT).

\section{INTRODUCTION}

Stress is a huge problem in today's society. Stress may occur due to several factors, including the working environment, economic background, health concerns, family issues and involvement in stressful situations, such as examinations, violent confrontations and road traffic [1]. Excessive exposure to the prolonged stress may lead to the depression and insomnia. Thus, the negative implications due to the stress may affect the quality of life.

The stress causes the abnormality in body regulation due to the excessive or insufficiency of hormone production. Most of body system and body regulation are governed by brain. The interaction in brain between neurons generates electrical

Manuscript received March 20, 2014; revised May 16, 2014. This work was supported in part by the Universiti Malaysia Perlis.

Saidatul A. is with the Intelligent Signal Processing Cluster, Universiti Malaysia Perlis, 02600, Arau, Perlis, Malaysia (e-mail: saidatul@ unimap.edu.my)

Paulraj Murugesa Pandiyan and Sazali Yaacob are with School of Mechatronics, University Malaysia Perlis, 02600, Arau, Perlis, Malaysia (e-mail: paul@unimap.edu.my, sazali22@yahoo.com.my). activity. These electrical activities are captured by Electroencephalography (EEG) as a brain signals. EEG is the one of the most common method used to study the brain function and brain condition. However, EEG signal is highly random, non linear and highly complex signals; thus, advanced signal processing technique is needed to extract the mental stress features.

In mental stress study, developing high quality database of EEG signals is the crucial part. It is desirable that the influence of mental stress on the central nervous system is directly reflected in the EEG signals. In collecting high quality database, it is not easy to judge whether the targeted stress state is properly induced or not. This is because, the response among the individual may be varies due to the demographic factors and initial condition of the subjects. There are few aspects that may cause the variation of the response such as gender, family background, natural behavior, profession and etc. This chapter argues a set of preliminary experiments conducted towards assessing the human mental stress.

The main objective of this paper is to propose the mental stress elicitation protocol and to validate the ability of designed protocol to elicit mental stress. Fig. 1 illustrates the overview of mental stress recognition system using EEG signal. Typically, mental stress recognition system consists of four steps; i) Data acquisition protocol ii) Pre-processing iii) Feature Extraction and iv) Classification.

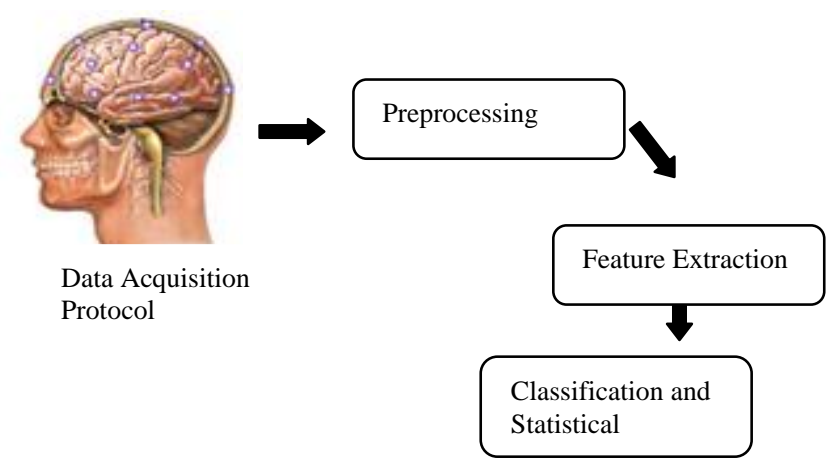

Fig. 1. Overview of mental stress recognition system.

Brief descriptions for each step as follows:

1) Data acquisition - Measuring brain activity effectively is a critical step for brain recognition system communication. Human response modulates the electrical signals which are measured using electrodes cap and then these signals are digitized. Mindset-24 Topographic Neuro-mapping Instrument by Nolan Computer Systems LLC along with an electrode cap is used. 
2) Pre-processing - The aim of this stage is to improve the quality of signal by reducing and discarding noise without losing information.

3) Feature Extraction - The brain patterns used in brain computer interface are characterized by certain features. Feature extraction aims at describing the signals by a few relevant values called 'features'[2].

4) Classification and Statistical Analysis - This step is proposed to validate the designed elicitation protocol. In this stage, three methods are employed such as kNN classifier, Alpha Brain Asymmetry Score and Paired T-test. The classification step assigns a class to a set of features extracted from the signals. This class corresponds to the type of mental states identifies. The result or output from those methods (paired t-test, kNN classifier and Alpha Brain Asymmetry Score) can be used as an indicator to prove the mental stress through designed protocol is properly induced.

The detailed experimental protocol of related works, signal processing method, validation protocol and experimental result and discussions is discussed in the next section.

\section{RELATED WORKS}

Most researchers induce and measure stress under laboratory conditions instead of a real life situation because of several limitations, including physical limitations, such as the unavailability of compact data acquisition devices, and procedural limitations, such as the complexity of the experimental protocols and the effects of noise and other confounding factors that inevitably encountered in an uncontrolled environment.

However, most researchers assume that the effect of stress induced in a laboratory environment on the Autonomous Nervous System (ANS) activity is very similar to the effect of real-time stress. Hence, laboratory based experiments continue to be widely used because it is easier to generate the requisite quantities of stress and baseline (relaxation) samples in a controlled environment. According to the human behavior and psychology, there are several ways to induce mental stress for human. The method that have been used for previous studies are summarized in Table I.

TABLE I: TyPE OF INDUCER THAT HAVE BEEN USED TO INDUCE MENTAL

\begin{tabular}{clll}
\multicolumn{3}{c}{ STRESS IN PREVIOUS STUDIES } \\
\hline Ref & Author & \multicolumn{2}{l}{ Method/Stimuli } \\
\hline 1 & Choi et al. & $\begin{array}{l}\text { Mental } \\
\text { Task }\end{array}$ & Arithmetic \\
& & Mental & Arithmetic \\
2 & Krantz et al. & Task & \\
& & Mental & Arithmetic \\
3 & Taelman et al. & Task & \\
& & Mental & Arithmetic \\
4 & W.Linden et al. & Task & \\
& & Mental & Arithmetic \\
5 & K.Yashima et al. & Task & \\
& & Driving Simulator \\
6 & Tran et al. & Driving Simulator \\
\hline
\end{tabular}

Based on the Table I, most of the studies were adopted mental arithmetic task as a mental stress inducer. This method is commonly used in psychology studies to induce mental stress in human [3]-[7]. Furthermore, other technique such as driving simulator has been adapted to study the changes in brain dynamics of stress and fatigue [8], [9].

Up to now, there are no standard methodologies in mental arithmetic task. The combination between four mathematical operations such as addition, subtraction, multiplication and division are always used in the mathematical arithmetic inducer. However, the paradigm of the experimental protocol based on MAT are varies from researchers to other researchers. They were designed their protocol based on their knowledge that could induce mental stress in optimal way. In this study, some modifications have been made for existing mental arithmetic protocol studies. Dynamical excitation approach within time frame was adopted in designing protocol to elicit different level/ intensity of mental stress.

\section{MATERIAL AND METHODS}

\section{A. Subjects}

There are 30 healthy subjects (15 male and 15 females) aged between 21 and 23 years old with the mean age of 22.4 years have participated in this experiment. All the subjects were engineering university students without any previous history of medical, neurological and psychiatric illness. All the selected subjects are right handed. Furthermore, few criteria are determined to ensure the standardization of the subjects. There are: i) non-smokers iii) Malay speakers iv) those with vision that corrected to normal v) no high blood pressure problem vi) no exposure to general anesthesia in the last year.

\section{B. Experimental Set $U p$}

\section{1) Electroencephalography (EEG)}

EEG signal were collected by using standard Mindset 24 Topographic Neuro Mapping Instrument. A 19 Electro cap channel was used to record brain signal. EEG signals were measured with the electrodes fixed on the scalp at 19 sites namely Fp1, Fp2, F7, F3, Fz, F4, F8, T3, C3, Cz, C4, T4, T5, $\mathrm{P} 3, \mathrm{Pz}, \mathrm{P} 4, \mathrm{~T} 6, \mathrm{O} 1$ and $\mathrm{O} 2$ according to the international 10-20 system as shown in Fig. 2. EEG signals were recorded by a digital EEG machine with a 16 bits A/D resolution. The sampling frequency was set to $256 \mathrm{~Hz}$ and the impedances were kept below $5 \mathrm{k} \Omega$. The cutoff range signal was set as -80 to $80 \mu \mathrm{V}$. In this experiment, two computers were used simultaneously (Fig. 3). A computer was used to display mental arithmetic questions in order to elicit mental stress of the subjects, while the other to record the EEG signals of the subject during experiment session.

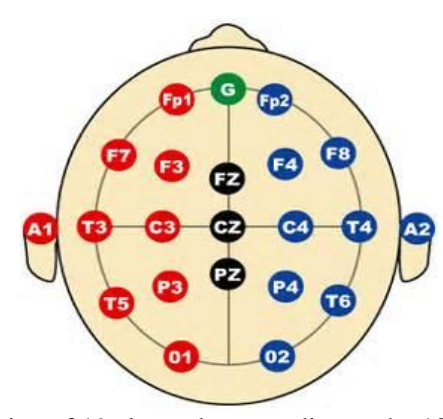

Fig. 2. The position of 19 electrodes according to the 10-20 international system. 


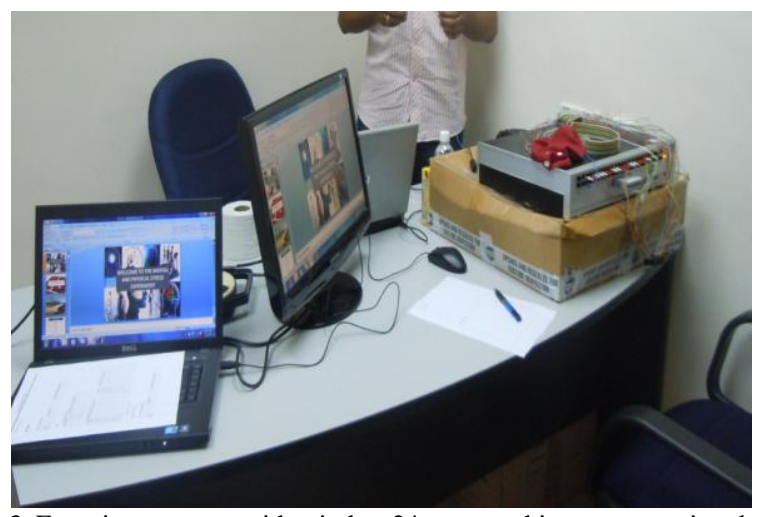

Fig. 3. Experiment set up with mindset 24 topographic neuromapping device and 2 desktops.

\section{Patient Monitoring System}

Patient Monitoring System (Fig. 4) is able to display blood pressure and pulse rate readings simultaneously. Blood pressure was measured three times from the right arm with a patient monitoring system (Patient Monitoring System, GE, German). Blood pressure (BP) at the right arm was measured in sitting position before and after experimental procedures.

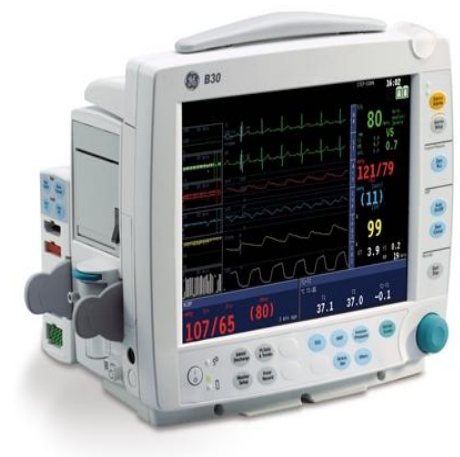

Fig. 4. Patient monitoring system.

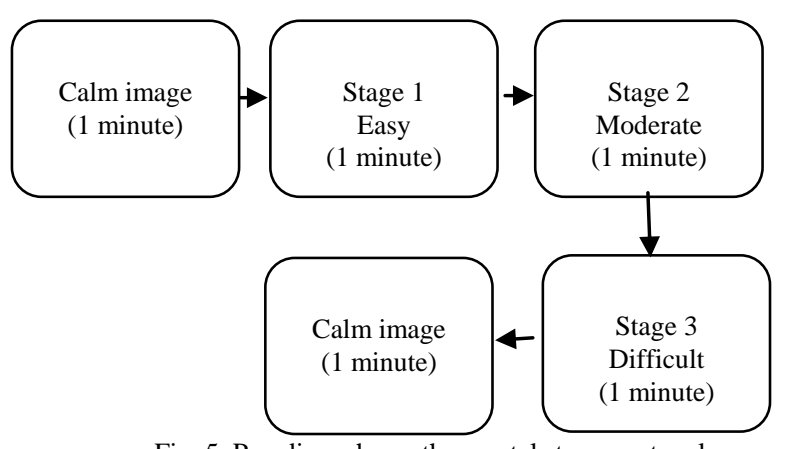

Fig. 5. Paradigm shows the mental stress protocol.

\section{Experimental Protocol}

The Mental Arithmetic Task (MAT) was displayed to subject in power point format. The subjects were asked to answer all the questions on a piece of paper. In order to reduce the artifact or noise, the subjects were asked to minimize the movement. Excessive head and eye movement is prohibited. The paradigm of the procedure is shown in Fig. 5. Basically, this experiment takes 5 minutes per session. Every subject was asked to repeat the same procedure twice in a day.

These sessions will be held in morning at 9 am and the other session at $2 \mathrm{pm}$ in the same day. These two sessions ( 9 am and $2 \mathrm{pm}$ ) were selected due to the resting time after an hour taking breakfast ( 8 am) and lunch meal (1 pm). Thus, it was assumed that the subjects were in well prepared and ready to start the experimental session. The paradigm shows that the procedure begins at calm image I followed by Easy stage, Moderate Stage and Difficult stage and the session was ended by calm image II. Each stage will last for one minute.

During mental arithmetic task, 12 questions will be given for every stage with average of 5 seconds per questions. Each stage involves three combinations of mathematical operations such as 4 questions for addition problems, 4 questions for subtraction problems and 4 questions for multiplication problems.

Every stage is differentiated based on the easy, moderate and difficult. The sample question for each stage is presented as follows:

1) Stage 1 (easy) - In this stage, the questions involves the single integer between 1 to 9 . Example: $2+2,4+5,1+1$, $2+3,9-3,4-2,5-1,8-4,2 \times 3,2 \times 1,4 \times 2,1 \times 9$

2) Stage 2 (moderate) - The questions involve the two integers between 10 to 99 . Example: $22+14,44+5$, $21+10,12+13,13-3,24-12,15-11,18-14,12 \times 3,15 \times 4$, $14 \times 2,13 \times 4$

3) Level 3 (Difficult) - The questions involve the three integers between 100 to 999. Example: $420+42,243+24$, $219+111, \quad 192+130, \quad 430-203, \quad 240-110, \quad 150-102$, $589-145,155 \times 20,225 \times 4,182 \times 5,331 \times 4$

\section{E. EEG Signal Processing}

The raw EEG data is contaminated with non cerebral source signal due to the movement, breathing, electrical interference known as artifact. The presence of artifacts may affect further signal processing in term of feature extraction and classification stage. During EEG recording, subjects generate artifacts like eye blinks and body movements, and other sources of artifacts are due to the electrical interferences and types of leads and electrodes.

In this study, the highest output value has been set as $80 \mu \mathrm{V}$ in the Mindset-24 instrument. Since the signal corresponding to the eye blink artifact is $100 \mu \mathrm{V}$, the eye blink has been eliminated from the EEG output signal. Hence, in the experiment analysis, no separate process was carried out to remove the eye blink artifacts. An Elliptic band pass filter were applied to extract the signal into four sub frequency bands namely delta $(1-4 \mathrm{~Hz})$, theta $(4-7 \mathrm{~Hz})$, alpha $(7-13 \mathrm{~Hz})$ and beta (13-30 Hz).

From the EEG spectrum, activity can be observed in the alpha band, where increased activity is related to increased relaxation (decreased stress) [10]-[12]. Thus, in this study, only alpha band is considered for further analysis.

\section{1) Preprocessing}

It is a standard practice to discard EEG into sub-frequency namely theta, delta, alpha and beta by way of a elliptic band pass filter [13]. The EEG signals from each stage (easy, moderate and difficult) were segmented into 60 segments with length of 1 second, so each EEG segment was 256 data points (samples in length). On the other hand, other researchers have suggested the used of elliptic filter for EEG signal classifications under mental activity. [14], [15]. Elliptic filter was used because of its low order as compared to other FIR filters like Butterworth. Forward and backward 
filtering was performed to ensure that there would be no phase distortion. Fig. 6 shows the sample of raw EEG signal has been filtered into alpha, beta, theta and gamma by using elliptic band pass filter.

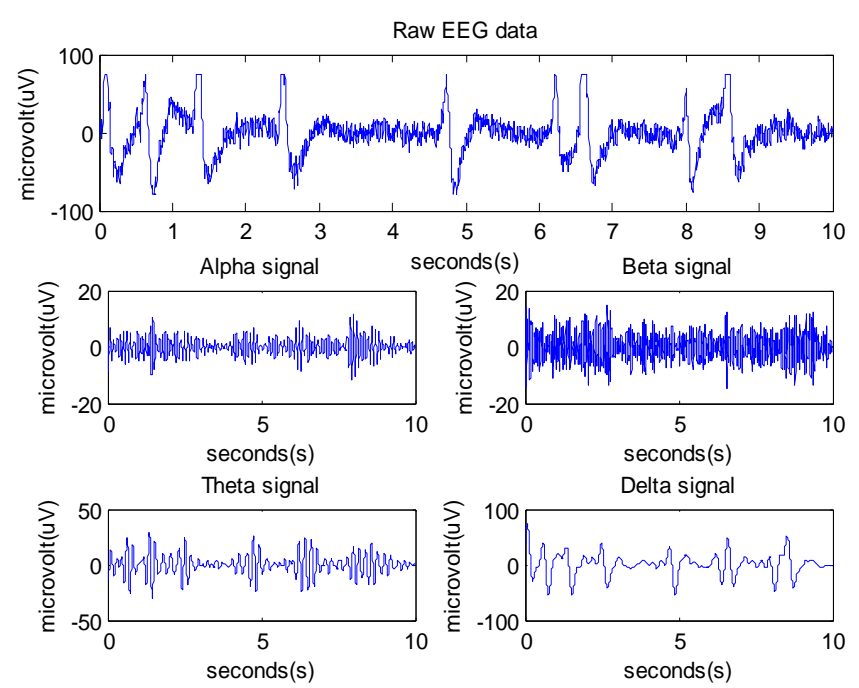

Fig. 6. Plots showing raw EEG signal and elliptic bandpass filtered EEG.

In this study, the segmented signals were filtered using $4^{\text {th }}$ order pass band elliptic filter and the setting of the band pass frequencies is 8 to $13 \mathrm{~Hz}$ [16]. Orders 4 were sufficient to obtain a $1 \mathrm{~Hz}$ stop-band beyond the pass-band on both sides. The filtered signals have only alpha waves so this means that undesired frequencies (such as spikes) have been rejected. The filter specifications were: $30 \mathrm{~dB}$ minimum attenuation in the stop-band with $0.5 \mathrm{~dB}$ ripple in the pass-band.

\section{2) Normalization}

Normalization (dividing each signal by the sum of the components within the signal) is carried out to reduce the effect of individual differences due to the fundamental frequency rhythms and also to reduce the computational complexity. All values of the attributes are normalized to lie in a common range of zero to one. The formula for rescaling between 0 to 1 is indicated in (1) as follows:

$$
Z_{i}=X_{i}-\min (x) /(\max (x)-\min (x)
$$

where is $x=$ the input data $\left(x_{1}\right.$ number, $Z_{i}=i^{\text {th }}$ normalized data

\section{3) Feature extraction using modified covariance}

Signal can be analyzed in term of time and frequency domain. In certain cases, the frequency domain of the signals is more useful and carries more information than the time domain characteristics. In the EEG studies, the frequency domain is able to show much richer information and it has been proved in the previous study with the classification accuracy more than $90 \%$ [17].

Thus, in this study, modified covariance was employed in order to extract the features from the mental stress (low, moderate and high) during mental arithmetic task. In modified covariance, no window is applied to the data, used for estimating Autoregressive (AR) model parameters.

Power Spectral Density value using modified covariance method is obtained using the built in function which is available in MATLAB signal processing toolbox. The built in function for Power Spectral Density as stated in (2):

$$
\left[P_{x x}, f\right]=\operatorname{pmcov}(x, p, n f f t, f s)
$$

where $x=$ input sequence, $p=$ number of order, $n f f t=$ length of the FFT to perform on each segment of data (default value is the minimum of 256 and the length of ' $x$ ') and fs= frequency sampling. $P_{x x}$ returns the power spectrum of the input signal. If the $x$ is real, the length of $\mathrm{P}_{\mathrm{xx}}$ for an even and for an odd $n f f t$ is given by (3) and (4) below. If $x$ is complex, the length of $P_{x x}$ is nfft. fs returns the frequencies that correspond to $P_{x x}$.

$$
\begin{gathered}
n f f t / 2+1 \\
(n f f t+1) / 2
\end{gathered}
$$

\section{F. Feature Reduction using Statistical Features}

In this stage, two statistical features are selected to represent mental stress features, i.e: power and energy. The power and energy for Alpha band are used for classifying the human mental stress from EEG signal. They are two statistical features called:

1) EEG frequency band power - This statistical feature is one of the most useful features for assessing the information content on the EEG signals[14]. The frequency band power is calculated by (5):

$$
P_{x}=\frac{1}{N} \sum_{n=k}^{k+N-1}|x[n]|^{2}
$$

where $x[n]=$ segmented EEG signal, $\mathrm{N}=$ data length of the signal.

2) EEG frequency band energy - the energy of the EEG can be partitioned at different levels in different ways depending on the frequency bands. It remains unaffected by the duration of the mental stress elicited. The frequency band energy is calculated by (6):

$$
E_{x}=\frac{1}{N} \sum_{n=-\infty}^{\infty}|x[n]|^{2}
$$

where $x[n]=$ segmented EEG signal and its units are simply the square of the units of the signal itself.

\section{G. Signal Analysis}

\section{1) K-nearest neighbors $(\mathrm{kNN})$}

KNN classifier is the famous and simple classifier that commonly used by the researchers. It is a powerful technique for pattern classification of nonparametric analysis. In this classifier, the testing data has been compared with the training data (baseline data). This classifier finds the value of $k$ in the neighbourhood of training data and the data will be assigned based on the more frequently data appeared in the neighbourhood of $k$. In this study, $k$ values are varied from 1 to 10 . The variation of $k$ value is important to find the match class between testing and training data.

The default setting of this KNN classifier was set to 'Euclidean' and 'Nearest'. The equation of 'Euclidean' distance is shown in (7) as follows: 


$$
D_{E}(a, b)=\sum_{i=1}^{N} \sqrt{\left(a_{j}-b_{k}\right)^{2}}
$$

where $D_{E}=$ distance, $N=$ Number of features, $a_{j}$ or $b_{k}=$ either testing or training data.

\section{2) Statistical analysis using paired t-test}

Paired t-test is the most common used to statistical method to analyze the study based on 'pre-post' design. A study of this type often consists of two measurements taken on the same subject, one before and one after the introduction of a treatment or a stimulus. In this study, the mean of systolic/diastolic blood pressure and pulse rate are measured before and after experimental procedure. In this case, mental arithmetic task is a treatment or a stimulus.

The basic idea is explained as follows: If the treatment had no effect, the average difference between the measurements is equal to 0 and the null hypothesis holds. On the other hand, if the treatment did have an effect, the average difference is not 0 and the null hypothesis is rejected. The equation for paired t-test is shown in (8) as follows:

$$
t=\frac{\sum d}{\sqrt{\frac{n\left(\sum d^{2}\right)-\left(\sum d\right)^{2}}{n-1}}}
$$

where $n$ is a number of data and $d$ is the differences between the values of each pair, pre and post. For significance tests, a two tailed $p$ value with threshold of 0.05 or less was considered statistically significant. In this study, this test was used to measure the significance of the changes in blood pressure and pulse rate for pre and post with respect to the stimulus. Hence, the stimulus or MAT is assumed may give effect to the blood pressure and pulse rate if the significance value is less than 0.05 .

\section{3) Alpha brain asymmetry score}

There is an abundance on evidence supporting the frontal cortex is particularly critical in emotion processing from prefrontal EEG alpha asymmetry studies[18]-[20]. Specifically, positive moods or reactions have been found to predict relatively greater left prefrontal activity, whereas negative mood (stress) have been found to predict relatively greater right prefrontal activity [21]. Asymmetry scores represented the difference between log alpha density in the right hemisphere electrodes of interest and log alpha density in the left hemisphere electrodes of interest or $\ln (\mathrm{R} / \mathrm{L})$ alpha power [22]. The formula for Alpha Asymmetry Score (AAS) is presented in (9) as follows:

$$
\text { Alpha Asymmetry Score }(\mathrm{AAS})=\ln \left[P_{x}\right]_{R}-\ln \left[P_{x}\right]_{L}
$$

where $P_{x}$ is Alpha band power as stated in (5). R refers to right hemisphere electrodes and $L$ refers to left right hemisphere electrodes.

\section{RESUlTS AND DiscUSSIONS}

\section{A. Validation based on kNN classifier}

For the validation purposes, two statistical values (energy and power) from 2 channels (F3 and F4) were considered.
There are 12 trials per mental state and resulting of 4992 features from 26 subjects for 4 mental states (baseline, low, moderate, high). In this work, $70 \%$ of the samples were used to train the classifier and the remaining $30 \%$ were used for testing. Hence, from the total of 4992, 3494 (834 per mental state) were used for training and 1498 were used for testing.

TABLE II: CLASSIFICATION ACCURACIES OF STATISTICAL (ENERGY AND POWER) FEATURES USING KNN CLASSIFIER

\begin{tabular}{ccccccc}
\hline Features & $\boldsymbol{K}$ & Baseline & Low & Moderate & High & Average \\
\hline & 1 & 80.67 & 84.68 & 84.97 & 80.71 & 82.76 \\
& 2 & 80.15 & $\mathbf{8 5 . 5 6}$ & 83.62 & 81.44 & 82.69 \\
& 3 & $\mathbf{8 4 . 7 0}$ & 84.04 & 84.12 & 80.23 & 83.27 \\
Statistical & 4 & 81.51 & 84.34 & $\mathbf{8 5 . 0 0}$ & 82.09 & 83.23 \\
features & 5 & 81.48 & 83.16 & 82.86 & 81.34 & 82.21 \\
& 6 & 81.66 & 83.53 & 83.24 & 80.70 & 82.29 \\
& 7 & 82.34 & 84.99 & 83.39 & 80.84 & 82.89 \\
& 8 & 83.24 & 83.99 & 84.29 & $\mathbf{8 3 . 6 2}$ & $\mathbf{8 3 . 7 9}$ \\
& 9 & 80.13 & 85.70 & 84.45 & 82.70 & 83.24 \\
& 1 & 80.12 & 83.22 & 82.65 & 80.66 & 82.43 \\
\hline
\end{tabular}

Table II shows the classification accuracies of statistical features (power and energy) using KNN classifier. The average classification accuracy for four mental states is $83.79 \%$. The statistical features show a consistent average accuracy for the different values of $\mathrm{k}$. Based on the average percentage of accuracies which is above $80 \%$ for all mental states, it clearly shows that the designed experiment is likely works to elicit the mental stress with different level intensity of stress. The percentage of above $80 \%$ is acceptable where most of the research related to the EEG signal classification considers the system is reliable with the classification accuracy above $80 \%$ [23]-[25].

\section{B. Validation Based on Statistical Analysis}

Autonomic Nervous System (ANS) is divided into two main systems namely sympathetic nervous system (SNS) and parasympathetic nervous system (PNS). The SNS is responsible for regulation of internal organ which occurs unconsciously. SNS occurs when the body is at rest such as sleeping, eating and etc. Nevertheless, PNS is responsible for stimulating activities associated with the fight-or-flight response.

Hence, respiratory system and blood circulatory will be affected due to the demand of the oxygen supply to body tissues. The physiological changes that can be monitored due to the PNS responds are heart rate and blood pressure. Table III shows the paired t-Test result for pre and post experiments of blood pressure and heart rate for all subjects.

TABLE III: PAIRED T-TEST RESUlTS FOR DIFFERENT BETWEEN PRE AND POST EXPERIMENTS OF BLOOD PRESSURE AND HEART BEAT

\begin{tabular}{lcccc}
\hline & $\begin{array}{c}\text { Difference } \\
\text { (post-minus } \\
\text { pre study) }\end{array}$ & $\begin{array}{c}\text { Standard } \\
\text { Deviation of } \\
\text { difference }\end{array}$ & $\boldsymbol{T}$ & $\boldsymbol{P}$ \\
\hline SBP $(\mathbf{m m ~ H g})$ & 5.1 & 13.45 & 3.8 & 0.01 \\
DBP(mm Hg) & 4.5 & 18.30 & 2.6 & 0.05 \\
Heart rate & 13.9 & 7.9 & 5.4 & $<0.001$ \\
(beats/min) & & 7.9 & & \\
\hline
\end{tabular}

$\overline{\mathrm{SBP}}=$ Systolic blood pressure, $\mathrm{DBP}=$ Diastolic Blood Pressure, $\mathrm{SD}=$ Standard Deviation 
The average pre study systolic blood pressure (SBP) was $80 \pm 10 \mathrm{mmHg}$ and diastolic blood pressure was $70 \pm 8 \mathrm{mmHg}$. The average post study SBP was $120 \pm 8 \mathrm{mmHg}$ and DBP was $75 \pm 8 \mathrm{mmHg}$. The average pre study heart rate was $60 \pm 10$ beats/min and $90 \pm 8$ beats/min for the post study heart rate. Paired t-test analysis was performed on the pre study and post study blood pressure and heart rate. Table III shows that SBP and heart rate significantly increased $(p<0.05)$ after performing MAT. It clearly shows that the designed protocol was successful as it was able to elicit mental stress through MAT.

The findings of the current study are consistent with those of Liu et al. (2011) who found that blood pressure and heart rate is increased during stress state and indices appears to be an effective method by which to evaluate the influence of mental stress [26].

\section{Validation Based on Alpha Brain Asymmetry Score}

In order to investigate the ability of designed protocol to elicit the target emotion (stress), the concept of Prefrontal Alpha Brain Asymmetry is used. The effect of mental stress on alpha activity in the prefrontal brain region will indicate the state of the brain either under stress or not. The weighted averaged $\log$ alpha power values for anterior superior namely F3 and F4 were calculated. The left and right anterior superior quadrants contained the commonly analyzed dorsal F3/F4, respectively. Mean and standard deviation for the anterior superior quadrants during relaxation, low stress, moderate stress and high stress are presented in Table IV.

TABLE IV: MEANS OF Weighted AVERAge NATURAL Log AlPHa POWER EEG VALUES BY RIGHT AND LEFT QUADRANTS DURING RELAXATION, LOW

\begin{tabular}{|c|c|c|c|c|c|c|c|c|}
\hline \multirow{3}{*}{$\begin{array}{l}\text { Quad- } \\
\text { rant }\end{array}$} & \multicolumn{6}{|c|}{ STRESS, MEDIUM STRESS AND HIGH STRESS } & \multirow{2}{*}{\multicolumn{2}{|c|}{ High Stress }} \\
\hline & \multicolumn{2}{|c|}{$\begin{array}{l}\text { Baseline } \\
\text { (relaxation) }\end{array}$} & \multicolumn{2}{|c|}{ Low Stress } & \multicolumn{2}{|c|}{$\begin{array}{c}\text { Medium } \\
\text { Stress }\end{array}$} & & \\
\hline & $\mathrm{L}$ & $\mathrm{R}$ & L & $\mathrm{R}$ & $\mathrm{L}$ & $\mathrm{R}$ & $\mathrm{L}$ & $\mathrm{R}$ \\
\hline $\begin{array}{l}\text { Anterior } \\
\text { Superior }\end{array}$ & Mean & Mean & Mean & Mean & Mean & Mean & Mean & Mean \\
\hline F3-F4 & 0.21 & 0.23 & 0.21 & 0.22 & 0.19 & 0.21 & 0.19 & 0.18 \\
\hline
\end{tabular}

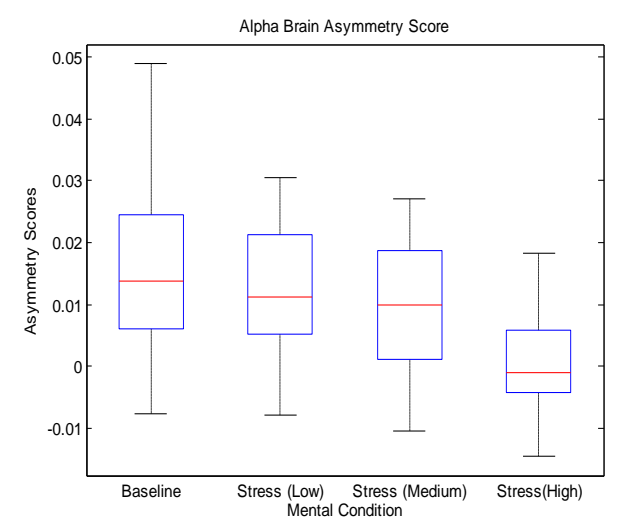

Fig. 7. Boxplot elucidates Alpha Brain Asymmetry Score for channel F3-F4 in four mental states; baseline (relaxation), low stress, medium stress and high stress.

Fig. 7 presents the results obtained from four mental states namely baseline, low stress, medium stress and high stress. The box plot (Fig. 7) shows some of the main characteristic of the asymmetry score (mean and standard deviation) to the mental conditions. There was a significant positive correlation between alpha asymmetry score and mental states. It is clear trend shown the mean averaged log alpha power between right and left hemisphere is decreased from relaxation to low stress, followed by moderate stress and high stress.

The activity of alpha becomes greater in right hemisphere during relaxation or low stress and slowly disrupted when it is shifted to higher level of stress. The present findings seem to be consistent with other research which found the a shift from relatively greater left frontal activity during low stress to relatively greater right frontal activity during high stress [21].

\section{CONCLUSIONS}

By comparing three validation techniques (Paired T-test, Alpha brain asymmetry and kNN classifier) in authenticating the designed protocol, it can be summarized mental stress influences the physiological changes of heart rate and EEG signals. These statements are supported by:

- Physiological changes based on blood pressure and heart rate indicates that the designed experimental protocol enables to elicit mental stress. The t-Test result showed that blood pressure and heart rate are significantly different ( $p$ value $<0.05$ ) during pre and post study.

- Alpha brain asymmetry in F3 \& F4 - it is shown that average mean values in four mental states namely baseline (relaxation), low stress, medium stress and high stress are comparable and have a potential to discriminate between these four conditions.

- The classification accuracy of approximately $84 \%$ using $\mathrm{kNN}$ classifier confirmed that proposed protocol have potential in classifying the mental stress level.

Thus, it can be concluded that developed experimental protocol based on MAT is able to emanate stress features by assessing the physiological changes of heart rate and EEG signal.

\section{REFERENCES}

[1] P. Rowden, G. Matthews, B. Watson, and H. Biggs, "The relative impact of work-related stress, life stress and driving environment stress on driving outcomes," Accident; Analysis and Prevention, vol. 43, no. 4, pp. 1332-40, Jul. 2011.

[2] R. K. B. G. E. Bashashati, A. Fatourechi, and M. Ward, "A survey of signal processing algorithms in brain-computer interfaces based on electrical brain signals," Journal of Neural Engineering, vol. 4, no. 2, pp. 35-57, 2007.

[3] J. Choi and R. Gutierrez-osuna, "Using heart rate monitors to detect mental stress," Stress: The International Journal on the Biology of Stress, pp. 221-225, 2009.

[4] G. Krantz, M. Forsman, and U. Lundberg, "Consistency in physiological stress responses and electromyographic activity during induced stress exposure in women and men," Integrative Physiological and Behavioral Science: The Official Journal of the Pavlovian Society, vol. 39, no. 2, pp. 105-118, 2004.

[5] J. Taelman, S. Vandeput, A. Spaepen, and S. Van Huffel, "Influence of mental stress on heart rate and heart rate variability," Heart, pp. 1366-1369, 2009.

[6] W. Linden, "What do arithmetic stress test measure?" Psychophysiology, vol. 28, no. 1, pp. 91-102, 1991.

[7] K. Yashima, T. Sasaki, Y. Kageyama, M. Odagaki, and H. Hosaka, "Application of wavelet analysis to the plethysmogram for the evaluation of mental stress," in Proc. Annual International Conference of the IEEE Engineering in Medicine and Biology Society, Jan. 2005, vol. 3, pp. 2781-2784.

[8] Y. Tran, R. A. Thuraisingham, N. Wijesuriya, H. T. Nguyen, and A. Craig, "Detecting neural changes during stress and fatigue effectively: 
a comparison of spectral analysis and sample entropy," in Proc. 2007 3rd International IEEE/EMBS Conference on Neural Engineering, May 2007, pp. 350-353.

[9] M. Jiang and Z. Wang, "A method for stress detection based on FCM algorithm," in Proc. 2009 2nd International Congress on Image and Signal Processing, Oct. 2009, pp. 1-5.

[10] S. Reisman, "Measurement of stress," in Proc. the IEEE 23rd Northeast, Bioengineering Conference, 1997, pp. 21-23.

[11] D. Mathersul, L. M. Williams, P. J. Hopkinson, and A. H. Kemp, "Investigating models of affect: Relationships among EEG alpha asymmetry , depression , and anxiety," Emotion, vol. 8, no. 4, pp. 560 $-572,2008$.

[12] S. Sridhar, "Estimation of effects of alpha music on EEG components by time and frequency domain analysis," in Proc. International Conference on Computer and Communication ENginerring, May 2010, pp. 11-13.

[13] M. M. Shaker, "EEG waves classifier using wavelet transform and fourier transform," International Journal of Biomedical Sciences, pp. 723-728, 2007.

[14] R. Palaniappan, "Brain computer interface design using band powers extracted during mental tasks," in Proc. 2nd International IEEE EMBS Conference on Neural Engineering, 2005, pp. 321-324.

[15] R. Palaniappan and D. P. Mandic, "EEG based biometric framework for automatic identity verification," The Journal of VLSI Signal Processing Systems for Signal, Image, and Video Technology, vol. 49, no. 2, pp. 243-250, Jun. 2007.

[16] R. Palaniappan, "Electroencephalogram signals from imagined activities: A novel biometric identifier for a small population," pp. 604-611, 2006.

[17] E. D. Übeyli, D. Cvetkovic, and I. Cosic, "AR spectral analysis technique for human PPG, ECG and EEG signals," Journal of Medical Systems, vol. 32, no. 3, pp. 201-206, Jan. 2008.

[18] W. B. Kang, D. H. Davidson, R. J. Coe, C. L. Wheeler, R. E. Tomarken, and A. J. Ershler, "Frontal brain asymmetry and immune function," Behavioral Neuroscience, vol. 105, pp. 860-869, 1991.

[19] D. J. Davidson, R. J. Schwartz, G. E. Saron, C. Bennett, and J. Goldman, "Frontal versus parietal EEG asymmetry during positive and negative affect," Psychophysiology, vol. 16, pp. 202-203, 1979.

[20] B. Davidson, R. J. Coe, D. D. Dolski, and I. Donzella, "Individual differences in prefrontal activation asymmetry predict natural killer cell activity at rest and in response to challenge," Brain, Behavior and Immunity, vol. 13, pp. 93-108, 1999.

[21] R. S. Lewis, N. Y. Weekes, and T. H. Wang, "The effect of a naturalistic stressor on frontal EEG asymmetry, stress, and health.," Biological psychology, vol. 75, no. 3, pp. 239-247, Jul. 2007.

[22] J. L. Stewart, J. A. Coan, D. N. Towers, and J. J. B. Allen, "Frontal EEG asymmetry during emotional challenge differentiates individuals with and without lifetime major depressive disorder," Journal of Affective Disorders, vol. 129, no. 1-3, pp. 167-714, Sep. 2010.

[23] M. Murugappan, "Inferring of human emotional states using multichannel EEG," European Journal of Scientific Research, vol. 48, no. 2, pp. 281-299, 2010.

[24] S. Rezaei, K. Tavakolian, and K. Naziripour, "Comparison of five different classifiers for classification of mental tasks.," in Proc. Annиal International Conference of the IEEE Engineering in Medicine and Biology Society, Jan. 2005, vol. 6, pp. 6007-10.

[25] M. Sabeti, R. Boostani, S. D. Katebi, and G. W. Price, "Selection of relevant features for EEG signal classification of schizophrenic patients," Biomedical Signal Processing and Control, vol. 2, no. 2, pp. 122-134, Apr. 2007.

[26] X. Liu, K. Iwanaga, Y. Shimomura, and T. Katsuura, "Comparison of stress responses between mental tasks and white noise exposure," Journal of Physiological Anthropology, vol. 26, no. 2, pp. 165-171, 2007.

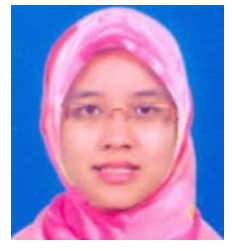

human stress.
Saidatul A. received the degree in biomedical engineering from University of Malaya in 2006. She took up his academic pursuit in 2007 as a lecturer in University Malaysia Perlis after completing her master program of Medical Physics, University of Malaya in a year. Currently, she is a PhD student at Intelligent Signal Processing, University Malaysia Perlis. Her area of specialization is signal processing in related to 